\title{
INVESTIGACIONES
}

\section{Una taxonomía de medios educativos para el desarrollo del pensamiento crítico: Dominios de acción y tipologías textuales}

\author{
A taxonomy of educational media for the nurture of critical thinking: \\ Action domains and text typologies
}

\author{
Aarón Elí Mena Araya \\ Escuela de Ciencias de la Comunicación Colectiva, Universidad de Costa Rica. \\ aaron.menaaraya@ucr.ac.cr
}

\section{RESUMEN}

El artículo se basa en el análisis de distintas aproximaciones a los objetos, procesos y enseñanza del pensamiento crítico, para proponer una taxonomía bidimensional. La primera dimensión se enfoca en las acciones generales y específicas relacionadas con los componentes centrales del pensamiento crítico, y plantea cinco dominios de acción: aplicación de lógica informal, resolución de problemas en las ciencias, resolución de problemas en la vida ciudadana, comprensión y creación de contenidos mediáticos y metacognición. La segunda dimensión ofrece un marco para la visualización sistematizada de las características formales de los distintos medios educativos que pueden apoyar el aprendizaje del pensamiento crítico, y se propone el uso de cinco tipologías textuales: narración, descripción, explicación, argumentación y diálogo. Adicionalmente se definen procedimientos concretos para la articulación de las dos dimensiones de la taxonomía en el análisis, clasificación y diseño de medios educativos dirigidos al desarrollo de habilidades de pensamiento crítico.

Palabras clave: pensamiento crítico, medios educativos, taxonomía, objetivos de aprendizaje, tipologías textuales.

\section{ABSTRACT}

This paper proposes a bidimensional taxonomy based upon the analysis of various approaches to the objects, processes and teaching of critical thinking. The first dimension focuses on the general and specific actions related to the main components of critical thinking and puts forth five action domains: application of informal logic, problem-solving in sciences, problem-solving in citizen life, creation and comprehension of media content, and metacognition. The second dimension offers a framework for the systematic visualization of the formal characteristics of the different educational media supporting the learning of critical thinking and proposes the use of five text typologies: narrative, description, explanation, argumentation and dialogue. Furthermore, the paper outlines specific procedures for the articulation of both dimensions of the taxonomy in the analysis, classification and design of educational media aimed to support the development of critical thinking skills.

Key words: critical thinking, educational media, taxonomy, learning objectives, text typology. 


\section{INTRODUCCIÓN}

La conceptualización, desarrollo e implementación de los currículos para la enseñanza del pensamiento crítico han sido campos relevantes en la investigación educativa desde que Dewey (1910) introdujo el término de pensamiento reflexivo hace poco más de un siglo. En años recientes el desarrollo del pensamiento crítico ha empezado a cumplir un rol cada vez más importante en todos los niveles educativos, a medida que los nuevos currículos incorporan los conocimientos, habilidades y actitudes necesarias para la ciudadanía global, la resolución de problemas y la literacidad mediática. Este proceso ha llevado a la creación y distribución de numerosos medios educativos dirigidos a apoyar no solamente la educación escolar, sino también la educación permanente.

La variedad y amplitud en la oferta de medios educativos presentan retos importantes para la comunidad docente. La selección de medios educativos pertinentes para el cumplimiento de objetivos de aprendizaje concretos requiere no solamente conocimientos básicos sobre la teoría y la práctica de la enseñanza del pensamiento crítico, sino también una comprensión profunda de la manera en que los medios educativos pueden apoyar, o en dado caso entorpecer, el aprendizaje de habilidades de pensamiento específicas. El personal docente y administrativo de los centros educativos, quienes trabajan bajo estrictas limitaciones de tiempo, y en muchos casos no cuentan con una formación exhaustiva en estos temas, pueden encontrar grandes dificultades para identificar y seleccionar, con confianza y consistencia, los medios educativos idóneos para apoyar los procesos de aprendizaje.

Por otra parte, el diseño de medios educativos implica el trabajo colaborativo de expertos en educación y en producción mediática. Usualmente, la experticia en estos campos es adquirida a través de rutas académicas y profesionales distintas, lo cual obliga a educadores y productores a trabajar juntos, conduciendo diálogos interdisciplinarios con el fin de encontrar espacios comunes, que les permitan crear medios educativos que además de contribuir al cumplimiento de objetivos de aprendizaje específicos, sean pertinentes desde un punto de vista comunicativo y estilístico.

El presente texto propone una taxonomía para el análisis y el diseño de medios educativos sobre pensamiento crítico, y tiene dos objetivos principales. Primeramente, busca ofrecer un marco para la identificación, clasificación y selección de medios educativos enfocados en el desarrollo del pensamiento crítico, que sea útil para docentes, autoridades administrativas e investigadores. Adicionalmente, busca brindar herramientas dirigidas al enriquecimiento del diálogo entre los educadores y productores que colaboran en la creación de dichos medios educativos.

\section{METODOLOGÍA}

La teoría y la práctica de la enseñanza del pensamiento crítico pueden ser abordadas desde una gran variedad de perspectivas. En este sentido, no hay un consenso final sobre qué puede ser considerado como pensamiento crítico o sobre en qué contexto y cómo debe ser enseñado. Esta diversidad de perspectivas también se manifiesta en los medios educativos relacionados con la promoción de las habilidades de pensamiento crítico. Por este motivo, como primer paso para la construcción de la taxonomía presentada en este artículo, se 
identificaron los componentes centrales del pensamiento crítico a través de una revisión de literatura publicada en inglés, japonés y castellano. Utilizando estos componentes como base, se propone la primera dimensión de una taxonomía bidimensional.

El siguiente paso consistió en la selección de un conjunto de categorías que permitieran la clasificación de diferentes tipos de medio según sus características formales y estructurales. Estas categorías fueron utilizadas para construir la segunda dimensión de la taxonomía. De manera adicional, se incluye una explicación sobre la manera en que las dos dimensiones de la taxonomía se relacionan entre sí, y sobre cómo la taxonomía puede ser aplicada para la clasificación y diseño de los medios educativos.

Finalmente, se seleccionaron y analizaron ejemplos concretos de medios educativos sobre pensamiento crítico, con el propósito de determinar la funcionalidad y validez de las dimensiones y los mecanismos presentados en la taxonomía. Como muestra se utilizaron cinco medios educativos, dados a conocer entre el 2011 y el 2018, mediante publicaciones académicas o través de librerías y repositorios electrónicos de medios de comunicación u organizaciones educativas.

\section{PENSAMIENTO CRÍTICO Y MEDIOS EDUCATIVOS}

\subsection{EL ROL DE LOS MEDIOS EDUCATIVOS EN LA ENSEÑANZA DEL PENSAMIENTO CRÍTICO}

La construcción de una taxonomía para la clasificación y diseño de medios educativos sobre pensamiento crítico requiere, primeramente, una comprensión de la manera en que los medios educativos se relacionan con el currículo en la educación escolar y con la educación permanente. Diversos modelos ofrecen una guía para la enseñanza del pensamiento crítico. Algunos proponen que el pensamiento crítico puede ser enseñado a través de una combinación estrategias cognitivas y emocionales (Paul, Binker \& Weil, 1990; Facione, 1990) o mediante la enseñanza de conjuntos de conocimientos, habilidades, actitudes, éticas y valores (Griffin, McGaw \& Care, 2012). Otros proponen taxonomías que se enfocan en listas de disposiciones y habilidades generales (Ennis, 2016) o enfatizan componentes disposicionales, prácticos y metacognitivos que pueden ser transferidos a través de distintos dominios de conocimiento (Halpern, 2003). Todos estos marcos de referencia coinciden en la noción de que el pensamiento crítico es un proceso complejo, en el cual diferentes componentes interactúan entre sí.

La escala de uso, los métodos de operacionalización y el nivel de efectividad esperada varía ampliamente para cada uno de los modelos mencionados. Sin embargo, todos ellos son reconocidos como herramientas útiles para comprender los componentes que conforman el pensamiento crítico y para guiar el diseño de currículos para su enseñanza. ¿Puede alguno de estos modelos multidimensionales ser directamente aplicado a la clasificación y análisis de ejemplos individuales de medios educativos? ¿Es posible operacionalizar todos los componentes de alguno de estos modelos en el diseño y desarrollo de medios educativos concretos? En este artículo se propone que dichas tareas no solamente demandan una gran cantidad de tiempo, sino que además son inoportunas por dos motivos fundamentales: 1) cualquier modelo puede carecer de componentes valiosos presentes en otros modelos, y 2) el amplio espectro de habilidades y actitudes manifestado en cualquiera de estos modelos se extiende más allá de las posibilidades de cualquier medio educativo específico. 
Los currículos escolares, como guías para la definición de objetivos de aprendizaje, contenidos y métodos de instrucción pueden efectivamente reflejar la complejidad de cualquier modelo de pensamiento crítico. En contraste, los medios educativos, vistos desde una perspectiva vigotskyana, tradicionalmente cumplen el rol de apoyar el currículo, funcionando como andamios en actividades de aprendizaje específicas. Los medios educativos normalmente cuentan con dimensiones mucho más limitadas, tanto en contenidos como en objetivos, que las de los currículos en los que se encuentran circunscritos. Por este motivo, cualquier medio educativo que pueda contribuir al desarrollo del pensamiento crítico dentro de las fronteras del currículo escolar, muy probablemente estará enfocado en uno de sus componentes o estará limitado a un subconjunto de dichos componentes.

Los medios educativos también pueden ser una herramienta para promocionar el pensamiento crítico en el contexto de la educación permanente. Debido a que estos medios son utilizados de forma casual y fragmentada, por audiencias amplias y heterogéneas, en la educación permanente es aún más importante ofrecer contenidos accesibles, enfocados en objetivos de aprendizaje debidamente delimitados.

Los puntos discutidos en este apartado sugieren la necesidad de definir los componentes centrales del pensamiento crítico, con el fin de clarificar las áreas concretas en las que los medios educativos pueden apoyar su enseñanza de manera efectiva. Una vez definidos dichos componentes, es posible proceder a la tarea de delinear un marco de referencia apropiado para el análisis y el diseño de medios educativos sobre pensamiento crítico.

\subsection{LOS COMPONENTES CENTRALES DEL PENSAMIENTO CRÍTICO}

La visión sobre los objetos, objetivos y procesos del pensamiento crítico varían ampliamente entre distintas disciplinas. Mientras la filosofía se enfoca en cómo los individuos utilizan el pensamiento lógico, el discurso y la argumentación para guiar sus propias creencias y acciones, la psicología investiga principalmente la manera en que los procesos de pensamiento inciden en la construcción de significado, la resolución de problemas y la metacognición (Lewis \& Smith, 1993, p. 132). Una visión adicional es ofrecida por las ciencias cognitivas, las cuales enfatizan la importancia de las estrategias cognitivas y las heurísticas en el desarrollo de procesos cognitivos regularmente utilizados en la vida cotidiana, tales como la comparación, la clasificación y la inferencia (Resnick, 1987, p. 29-30). Lewis y Smith (1993, p. 134) llaman la atención también sobre la existencia de definiciones de pensamiento crítico que pueden interpretarse como complementarias. Mientras algunas de estas definiciones sostienen que el pensamiento crítico se relaciona principalmente con la resolución de problemas, otras hacen énfasis en la evaluación de argumentos y la emisión de juicios de valor. También es posible reconocer definiciones que sugieren una interacción entre la resolución de problemas y la evaluación.

Algunas definiciones recientes incorporan nuevos elementos a la discusión sobre la naturaleza del pensamiento crítico. Dwyer, Hogan y Steward (2014, p. 72) definen el pensamiento crítico como un proceso que involucra análisis, evaluación, inferencia y juicios reflexivos, y en el cual se articulan la memoria de trabajo y la memoria a largo plazo, y se manifiestan funciones autorregulatorias de metacognición. Para Michita (2008, p. 61) el pensamiento crítico es un proceso de resolución de problemas, en el cual inciden factores actitudinales, como el escepticismo y la reflexión, habilidades como el pensamiento lógico y el pensamiento creativo, y conocimientos sobre tecnología, sobre 
los sesgos recurrentes en los procesos pensamiento, y sobre campos de conocimiento específicos. Rodríguez (2018, p. 72), por su parte, define el pensamiento, el lenguaje y la acción como los elementos ontológicos del pensamiento crítico, y afirma que es mediante la interacción de estos elementos que los individuos pueden adquirir las habilidades para actuar de manera racional y autorregular su propio pensamiento.

Las revisiones de literatura sobre las tendencias de investigación sobre pensamiento crítico también señalan la prevalencia de diversos abordajes. Higuchi (2012, p. 200) reconoce la persistencia de un abordaje desde la lógica, enfocado en escepticismo, juicios de valor, evaluación y toma de decisiones, y un abordaje desde la psicología, centrado en la resolución de problemas y la metacognición. Identifica además a la definición de los principios del pensamiento crítico, la enseñanza y la práctica del pensamiento crítico en las asignaturas escolares, y la literacidad mediática como los tres temas más recurrentes en la investigación sobre pensamiento crítico. Otras revisiones presentan al pensamiento crítico como un conjunto de habilidades útiles para la resolución de problemas en campos académicos y profesionales específicos, tales como las ciencias naturales (Santos, 2017; Siew \& Mapeala, 2016; Loaiza y Osorio, 2018), las matemáticas (Widyatiningtyas, Kusumah, Sumarno \& Sabandar, 2015) y las ciencias médicas (Carvalho et al., 2017; Chan, 2016; Hincapie, Ramos y Chirino, 2018).

Modelos recientes basados en el desarrollo de competencias, dirigidos a apoyar el diseño curricular en un escenario global, incluyen sus propias aproximaciones al pensamiento crítico. La definición y selección de competencias de la OCDE (DeSeCo) propone como una primera categoría de competencias el uso interactivo de herramientas. Resalta la importancia de la reflexión crítica en el uso interactivo del lenguaje, símbolos y textos, el uso interactivo de conocimientos e información, y la utilización interactiva de la tecnología (Rychen \& Salganik, 2003).

El modelo para la evaluación y enseñanza de las competencias para el siglo XXI (ATC21s) incluye, dentro del grupo de formas de pensar, dos competencias que buscan integrar las distintas visiones sobre el pensamiento crítico: "pensamiento crítico, resolución de problemas y toma de decisiones" y "aprender a aprender, metacognición". La primera aborda el razonamiento efectivo (inducción y deducción), el uso de sistemas de pensamiento (análisis, categorización, inferencia, evaluación, etc.), la resolución de problemas, la emisión razonada de juicios y la toma de decisiones (Binkley et al., 2012, p. 40). La segunda resalta la importancia de la comprensión por parte del individuo de sus métodos de aprendizajes preferidos, el manejo efectivo del aprendizaje propio, y la apreciación del aprendizaje como una actividad enriquecedora de la experiencia de vida (Binkley et al., 2012, p. 44). Este modelo incorpora la literacidad mediática, otro de los campos comúnmente relacionados con el pensamiento crítico, proponiendo las competencias de "Literacidad informativa" y "Literacidad en TIC" como componentes del grupo de herramientas para trabajar.

Kusumi y Michita (2015, p. 183) apuntan que el pensamiento crítico también puede ser comprendido como una interacción compleja entre diferentes literacidades. Estas incluyen la literacidad mediática, que se relaciona con el uso efectivo y seguro de la internet y las tecnologías digitales, y las literacidades relacionadas con la lectoescritura, las ciencias y las matemáticas. Adicionalmente incorpora la literacidad en ciudadanía, la cual articula las literacidades en ciencias, economía, leyes, salud y otros dominios de conocimiento. La literacidad en ciudadanía les brinda a los ciudadanos las herramientas que les permiten 
obtener la información que necesitan en su vida cotidiana, y tomar acciones correctas basadas en dicha información. Con la adquisición de esta literacidad se espera que los ciudadanos sean capaces de involucrarse en los procesos sociales con un profundo sentido de la responsabilidad, resolver problemas, mostrar interés en el sufragio, y emitir juicios éticos y morales. Finalmente, el pensamiento crítico incluye las literacidades académicas y de investigación, que son las utilizadas por investigadores y estudiantes de educación superior para comprender y crear conocimiento especializado.

Reflexiones adicionales sobre los componentes del pensamiento crítico pueden ser encontradas en sistematizaciones previas de materiales educativos relacionados con el desarrollo de habilidades de pensamiento. Nickerson (1984, p. 29) identifica cinco abordajes diferenciados a la enseñanza del pensamiento crítico. El primero se enfoca en procesos cognitivos como la comparación, la clasificación, la inferencia y la predicción. Usualmente estos son comprendidos como procesos básicos e indivisibles, que se pueden aplicar en una amplia variedad de contextos.

El segundo abordaje se encuentra orientado en las heurísticas, las cuales son estrategias para resolver problemas, que, si bien no son perfectas, tienen la posibilidad de dar resultados positivos. Las heurísticas se utilizan para enfrentar problemas complejos, que no se pueden resolver con procedimientos predeterminados. En este abordaje se enfatiza la manera en que los expertos resuelven problemas en sus áreas de experticia (Nickerson, 1984, p. 30). El tercer abordaje se centra en el pensamiento formal, desde la visión piagetiana del desarrollo cognitivo. Los materiales educativos que utilizan este abordaje buscan ayudar a los estudiantes a realizar la transición entre la etapa de operaciones concretas y la etapa de operaciones formales (Nickerson, 1984, p. 32).

El cuarto abordaje se concentra en la manipulación del lenguaje y los símbolos, y se sustenta en la premisa de que el pensamiento conlleva el uso de algún tipo de símbolo. Dichos símbolos pueden sustentar formas de comunicación verbal, como la escritura en prosa, y formas de comunicación no verbal, como las ecuaciones. El último abordaje enfatiza la enseñanza del pensamiento como un objeto de estudio en sí mismo, y propone que el aprendizaje sobre los procesos de pensamiento puede ayudar a mejorar las habilidades de los estudiantes. Los recursos educativos enmarcados en este abordaje usualmente se centran en la identificación de los errores recurrentes en la construcción de argumentos.

Resnick (1987) ofrece un marco alternativo para organizar los programas de aprendizaje dirigidos al desarrollo de habilidades de pensamiento, refiriéndose a ellas como habilidades de pensamiento de orden superior. Propone seis categorías para clasificar los distintos tipos de programas disponibles para todos los niveles educativos, desde la formación primaria hasta la educación superior.

La primera categoría agrupa los programas que involucran la resolución de problemas en espacios disciplinarios específicos. Estos programas son utilizados principalmente en la educación superior, en campos como las ciencias, la ingeniería y las matemáticas. La segunda categoría reúne a los programas concentrados en la obtención de habilidades generales para la resolución de problemas en la vida cotidiana.

La tercera categoría, denominada "estrategias de lectura y estudio", enfatiza la identificación de la estructura y significado de los textos, y la detección de errores dentro de ellos. Estas estrategias pueden aprovecharse para desarrollar habilidades generales de lectura, que pueden ser transferidas a través de distintos dominios de conocimiento 
(Resnick, 1987, p. 24). La siguiente categoría se centra en las habilidades de automonitoreo, tales como la vocalización de ideas y el trabajo colaborativo, las cuales permiten a los estudiantes comprender y guiar su propio pensamiento (Resnick, 1987, p. 27).

La quinta categoría aborda los componentes de la inteligencia, y cubre el aprendizaje de vocabulario, el razonamiento espacial, la resolución de problemas y las habilidades de pensamiento lógico. Se utiliza en educación secundaria y superior y se sugiere que es el abordaje más limitado de los seis, pues se concentra en los elementos recurrentes en las pruebas de inteligencia. La última categoría se denomina "lógica informal y pensamiento crítico", y trata de brindarle a los estudiantes estructuras de pensamiento que les permitan identificar y evitar falacias lógicas. Los programas ubicados dentro de esta categoría suelen incluir el análisis de argumentos y textos narrativos (Resnick, 1987, p. 29).

A pesar de que Nickerson y Resnick resaltan diferentes áreas de instrucción, sus clasificaciones muestran diversos puntos de coincidencia. Del mismo modo, ambas nomenclaturas comparten elementos centrales con las definiciones y modelos de pensamiento crítico presentados previamente en este apartado. Estas concurrencias sugieren que a pesar de que no existe un consenso completo entre educadores e investigadores, cualquier aproximación a la definición e instrucción del pensamiento crítico involucra uno o más de los siguientes componentes:

- Lógica informal: referenciada en Nickerson (1984), Resnick (1987), Paul et al. (1990), Facione (1990), Lewis \& Smith (1993), Halpern (2003), Michita (2008), Higuchi (2012), Binkley et al. (2012), Dwyer, Hogan \& Steward (2014), Ennis (2016), Rodríguez (2018).

- Resolución de problemas: referenciada en Nickerson (1984), Resnick (1987), Lewis \& Smith (1993), Halpern (2004), Michita (2008), Higuchi (2012), Binkley et al. (2012), Ennis (2016), Higuchi (2012), Kusumi y Michita (2015, 2016), Widyatiningtyas, Kusumah, Sumarno \& Sabandar (2015), Siew \& Mapeala (2016), Chan (2016), Santos (2017), Carvalho et al. (2017), Loaiza y Osorio (2018), Ramos y Chirino (2018).

- Literacidad mediática: referenciada en Nickerson (1984), Resnick (1987), Paul et al (1990), Facione (1990), Rychen \& Salganik (2003), Halpern (2003), Higuchi (2012), Binkley et al. (2012), Kusumi y Michita (2015, 2016), Ennis (2016).

- Metacognición: referenciada en Nickerson (1984), Resnick (1987), Lewis \& Smith (1993), Michita (2008), Higuchi (2012), Binkley et al. (2012), Dwyer, Hogan \& Steward (2014), Ennis (2016), Rodriguez (2018).

Estos componentes pueden servir como la base para determinar los objetos de estudio y los objetivos de aprendizaje a ser tratados en los medios educativos sobre pensamiento crítico. ¿Pero puede cualquier ejercicio de pensamiento lógico, cualquier intento de resolver un problema, cualquier uso de los medios de comunicación o cualquier reflexión sobre el pensamiento propio contribuir al desarrollo del pensamiento crítico? ¿Qué procesos cognitivos específicos están involucrados en el aprendizaje del pensamiento crítico, ya sea que este se focalice en uno de sus componentes, o aborde varios componentes de manera transversal? El concepto de habilidad de orden superior puede ayudar a clarificar estos puntos. 


\subsection{EL PENSAMIENTO CRÍTICO COMO UNA HABILIDAD DE ORDEN SUPERIOR}

Lewis y Smith (1993, p. 134) señalan que una definición comprehensiva de habilidades de orden superior puede contribuir a establecer una conexión entre las habilidades referenciadas como "resolución de problemas" en las ciencias y como "pensamiento crítico" en las humanidades. Ellos definen las habilidades de orden superior como el uso efectivo de información "para cumplir un objetivo o encontrar una posible respuesta en situaciones complicadas", como decidir qué creer o qué hacer, creando nuevos objetos o solucionando problemas no enmarcados en una rutina. Resnick (1987, p. 44) anota que el pensamiento de orden superior involucra actividades mentales que requieren no solamente esfuerzo y emisión de juicios de valor informados, sino también la aplicación de múltiples criterios al análisis de situaciones complejas. Swartz et al. proponen una visión similar sobre las habilidades de pensamiento y definen el pensamiento hábil como "la aplicación estratégica de habilidades de pensamiento apropiadas y hábitos mentales productivos, a medida que sean necesarios para el desarrollo de productos razonados, tales como decisiones, argumentos y otros productos analíticos, creativos y críticos" (2010, p. 1). Newman (1990, p. 44) establece una distinción clara entre el pensamiento de orden inferior, que puede ser expresado a través de la aplicación mecánica de procedimientos y fórmulas previamente adquiridas, y el pensamiento de orden superior, que requiere la interpretación minuciosa, el análisis y la manipulación de nueva información.

Las taxonomías enfocadas en la organización de los objetivos de aprendizaje ofrecen otras explicaciones sobre la distinción entre las habilidades de pensamiento de orden inferior y de orden superior. Krathwohl (2002, p. 215) afirma que en el aprendizaje distintos tipos de conocimiento conceptual, procedimental y metacognitivo interactúan con procesos cognitivos, los cuales incluyen la recordación, la comprensión, la aplicación, el análisis, la evaluación y la creación. Dentro de este modelo se sugiere que las habilidades de orden superior empiezan a manifestarse en el nivel de comprensión, en donde el contenido de los textos es determinado a través de clasificación, inferencia, ejemplificación y otros procesos cognitivos específicos. Éstos se manifiestan de manera aún más clara en los siguientes niveles.

Marzano y Kendall (2007) toman un abordaje distinto a los objetivos de aprendizaje, y proponen una taxonomía que se enfoca en la acción recíproca entre procesos mentales y diferentes dominios de conocimiento. Los procesos mentales son organizados en seis niveles: recuperación de información, comprensión, análisis, utilización de conocimiento, sistema metacognitivo y sistema interno. El uso de habilidades de orden superior se vuelve explícito en el nivel de análisis, el cual involucra clasificación, análisis, y razonamiento deductivo e inductivo. Adicionalmente, el nivel de utilización de conocimiento incorpora la toma de decisiones y la resolución de problemas mediante investigación y experimentación.

Para abordar los medios educativos centrados en la promoción del pensamiento crítico de forma clara y significativa es necesario construir una taxonomía enfocada en acciones, que además de estar relacionadas con uno o más de los componentes del pensamiento crítico previamente identificados, puedan ser ubicadas dentro del ámbito de las habilidades de orden superior. Estas acciones incluirían así formas de aplicación de pensamiento lógico, resolución de problemas, uso de información, interacción con medios y tecnología, y metacognición que requieran la activación consciente y la combinación efectiva de procesos cognitivos. Por otra parte, se propone excluir las acciones pasivas limitadas a recordar información o a la aplicación mecánica de procedimientos y fórmulas. 


\section{PROPUESTA DE UNA TAXONOMÍA BIDIMENSIONAL}

\subsection{LA PRIMERA DIMENSIÓN DE LA TAXONOMÍA: DOMINIOS DE ACCIÓN}

En este apartado se delinea la primera dimensión de una taxonomía bidimensional. En esta tarea se toman en consideración dos elementos: 1) la interpretación del pensamiento crítico como un cúmulo de habilidades de orden superior que operan dentro y a través de componentes identificables, y 2) la comprensión de que, en cualquier contexto social, el proceso y los resultados del aprendizaje solo pueden ser observados en acciones específicas, que requieren necesariamente la interacción entre individuos, y la interacción en entre los individuos y diferentes tipos de medios de comunicación.

Utilizando los componentes centrales del pensamiento crítico como guía es posible establecer cinco dominios generales en los cuales se espera que los individuos sean capaces de realizar acciones determinadas. En esta taxonomía esos dominios son denominados "dominios de acción". A diferencia de los componentes centrales, que representan conceptos generales recurrentes en la teoría y práctica del pensamiento crítico, los dominios de acción se encuentran redactados para reflejar los ámbitos de acción vinculados a esos componentes. De este modo, a partir del componente de "lógica informal" se plantea el dominio de "aplicación de lógica informal". Tomando en cuenta la heterogeneidad de sus objetos, para el componente de "resolución de problemas" se proponen dos dominios de acción: "resolución de problemas en las ciencias" y "resolución de problemas en la vida ciudadana". El dominio de "comprensión y creación de contenido mediático" aborda el componente de "literacidad mediática". Finalmente, el componente de "metacognición" se aborda en un dominio de acción homólogo. Esto debido a que si bien la metacognición es un proceso que se desarrolla en la mente del individuo, dicho proceso y sus resultados pueden expresarse a través de acciones visibles y concretas.

Para cada dominio de acción es posible definir un conjunto de "objetos" y "acciones esperadas". Los objetos son los temas sobre los cuales los individuos piensan y actúan. En cualquier dominio estos objetos pueden tratar un amplio rango de problemas, situaciones, ideas y dilemas, debido que no se encuentran limitados a los contenidos de ninguna asignatura escolar. Por otro lado, las acciones esperadas se refieren a las acciones generales que articulan las tareas individuales y específicas relacionadas con cada dominio. La realización adecuada de estas acciones puede interpretarse como una indicación de que una persona domina, aunque sea de manera parcial, los conceptos y procedimientos relacionados con el dominio de acción. De este modo, al definir las acciones esperadas es posible obtener información valiosa sobre cuáles son los objetivos de aprendizaje compatibles con cada uno de los componentes del pensamiento crítico. 
Tabla 1. Dominios de acción

\begin{tabular}{|c|c|c|}
\hline $\begin{array}{l}\text { Dominio de } \\
\text { acción }\end{array}$ & Objetos & $\begin{array}{c}\text { Acciones esperadas } \\
\text { (objetivos de aprendizaje) }\end{array}$ \\
\hline $\begin{array}{l}\text { Aplicación de } \\
\text { lógica informal }\end{array}$ & \begin{tabular}{l|} 
Temas abstractos \\
relacionados con \\
pensamiento lógico, \\
argumentación, \\
discurso, semántica, y \\
perspectivas \\
contrapuestas.
\end{tabular} & $\begin{array}{l}\text { Las acciones generales incluyen la aplicación de pensamiento } \\
\text { lógico a la evaluación de argumentos y la participación en } \\
\text { interacciones dialécticas (Johnson, 2014, p. 11). } \\
\text { Acciones más específicas pueden incluir la identificación de } \\
\text { falacias lógicas, y el uso de razonamiento deductivo e inductivo } \\
\text { en situaciones de la vida cotidiana. }\end{array}$ \\
\hline $\begin{array}{l}\text { Resolución de } \\
\text { problemas en } \\
\text { las ciencias }\end{array}$ & \begin{tabular}{|l|} 
Problemas concretos \\
dentro de los campos \\
de las ciencias \\
naturales, la ingeniería \\
y las ciencias médicas. \\
\\
\end{tabular} & $\begin{array}{l}\text { Las acciones generales involucran la identificación de los } \\
\text { supuestos, las metas y los obstáculos de un problema (Mayer, } \\
\text { 1992, p. 5), y el uso adecuado de información para resolverlo } \\
\text { (Csapó \& Funke, 2017, p. 20). } \\
\text { Las tareas específicas incluyen la selección y aplicación idóneas } \\
\text { de conocimientos conceptuales, procedimentales y declarativos, } \\
\text { según la naturaleza del problema. Las tareas relacionadas con el } \\
\text { método científico, la observación, las mediciones y la } \\
\text { experimentación pueden cumplir también un rol importante en } \\
\text { la resolución de problemas dentro de este dominio de acción. }\end{array}$ \\
\hline $\begin{array}{l}\text { Resolución de } \\
\text { problemas en la } \\
\text { vida ciudadana }\end{array}$ & \begin{tabular}{l|} 
Problemas \\
relacionados con la \\
vida familiar, escolar y \\
comunitaria, con el \\
consumo de bienes y \\
servicios, con la \\
participación social y \\
con los procesos \\
electorales.
\end{tabular} & $\begin{array}{l}\text { Esto dominio comparte sus acciones generales con el dominio } \\
\text { previo. Sin embargo, en cada uno de ellos dichas acciones se } \\
\text { enmarcan de manera distinta. Mientras que el dominio anterior } \\
\text { enfatiza la validez científica y competencia técnica, éste se } \\
\text { enfoca en la importancia de actuar con base en visiones sociales } \\
\text { y políticas balanceadas y bien informadas. } \\
\text { Las acciones específicas incluyen el uso oportuno de } \\
\text { conocimiento en salud, economía, ciencia, política, leyes y } \\
\text { otros campos (Kusumi y Michita, 2016, p. 10). }\end{array}$ \\
\hline $\begin{array}{l}\text { Comprensión y } \\
\text { creación de } \\
\text { contenido } \\
\text { mediático }\end{array}$ & $\begin{array}{l}\text { Temas relacionados } \\
\text { con las características } \\
\text { retóricas y formales de } \\
\text { los medios, y con la } \\
\text { influencia de los } \\
\text { medios sobre la } \\
\text { sociedad y el } \\
\text { individuo. }\end{array}$ & $\begin{array}{l}\text { Como las principales acciones esperadas se proponen la lectura } \\
\text { concienzuda de los contenidos mediáticos, así como la creación } \\
\text { planificada y efectiva de dichos contenidos. } \\
\text { Estas acciones generales involucran el uso de conocimientos } \\
\text { conceptuales, como aquellos relacionados con los conceptos } \\
\text { clave de la literacidad mediática (Ontario Ministry of Education, } \\
\text { 1989), y conocimientos procedimentales para la utilización de } \\
\text { dispositivos electrónicos y software especializado en la creación } \\
\text { de contenidos (Eisenberg, Lowe \& Spitzer, 2004). }\end{array}$ \\
\hline Metacognición & $\begin{array}{l}\text { Temas relacionados } \\
\text { con procesos } \\
\text { cognitivos y estrategias } \\
\text { cognitivas. }\end{array}$ & $\begin{array}{l}\text { Las acciones generales requeridas son la organización, } \\
\text { visualización y expresión del pensamiento propio. } \\
\text { Acciones más específicas requieren conciencia sobre el } \\
\text { pensamiento propio, monitoreo activo de los procesos cognitivos } \\
\text { propios, y aplicación de heurísticas (Hennessey, 1999). Al igual } \\
\text { que el anterior, las actividades dentro de este dominio } \\
\text { frecuentemente conllevan el uso de tecnologías digitales. }\end{array}$ \\
\hline
\end{tabular}

Fuente. Elaboración propia (2019). 
Abordar los componentes del pensamiento crítico desde sus correspondientes dominios de acción tiene dos méritos concretos. Primero, el enfocarse en los objetos y las acciones esperadas (objetivos de aprendizaje) permite distinguir claramente el rol específico que un medio educativo determinado puede cumplir en la enseñanza del pensamiento crítico, ya sea en la educación escolar o en la educación permanente. Además, al utilizar este enfoque se vuelve posible incursionar en el diseño y producción de medios educativos efectivos, a través de un entendimiento claro de los componentes del pensamiento crítico y las acciones específicas relacionadas con dichos componentes.

Cabe resaltar que los dominios de acción propuestos en esta taxonomía engloban acciones específicas que pueden realizarse de manera simultánea o independiente, y cuya relevancia y complejidad varían según el contenido y dinámica de las actividades de aprendizaje. Cada uno de los dominios trata con objetos, objetivos y procedimientos distintos, por lo cual, el abordaje de las acciones de un dominio determinado no está necesariamente supeditado al abordaje previo de alguno de los otros dominios. No parece haber una ruta objetiva para afirmar que un dominio es más importante que otro, ya que todos ellos brindan espacios complementarios para el desarrollo y la aplicación de habilidades de pensamiento de orden superior. Por estos motivos, la correlación entre los dominios de acción no debe ser comprendida como una jerarquía estática.

Por otra parte, es posible identificar patrones recurrentes, en los que algunos dominios cumplen la función de apoyar a otros (ver figura 1). Por ejemplo, al brindar estrategias para el análisis y la deducción, la aplicación de la lógica informal apoya la resolución de problemas. De manera similar, la metacognición puede enriquecer tanto a la aplicación de lógica informal como a la resolución de problemas. Adicionalmente, del mismo modo que la comprensión de contenidos mediáticos cumple un rol importante en la organización de la información necesaria para para los procesos de pensamiento lógico y resolución de problemas, los resultados de estos procesos pueden ser expresados mediante la creación de contenidos mediáticos.

Figura 1. Interacciones recurrentes entre los dominios de acción

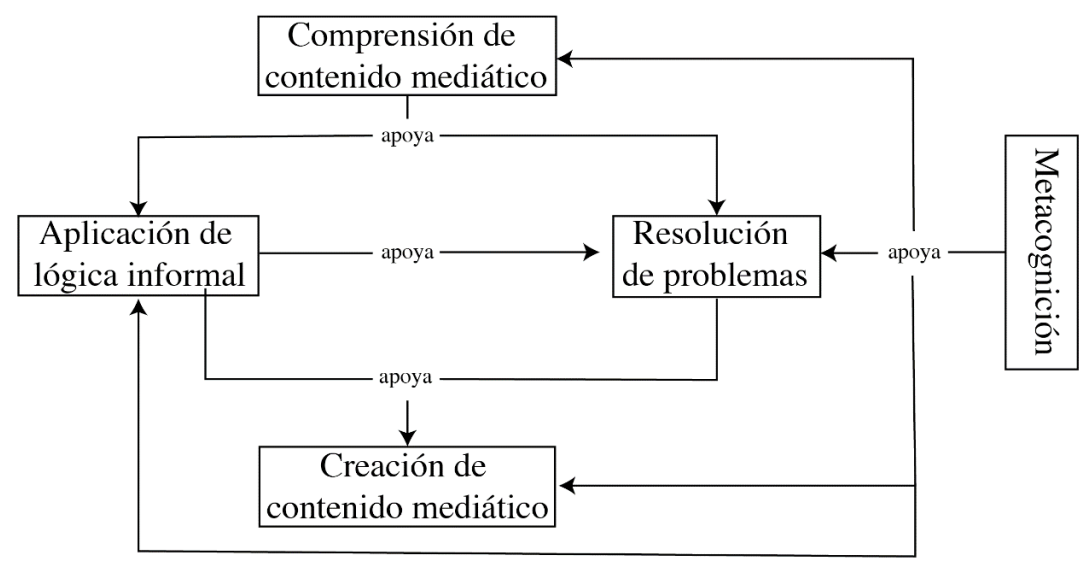

Fuente. Elaboración propia del autor (2019). 
Estudios Pedagógicos XLVI N 1: 203-222, 2020

UNA TAXONOMÍA DE MEDIOS EDUCATIVOS PARA EL DESARROLLO DEL PENSAMIENTO CRÍTICO: DOMINIOS DE ACCIÓN Y TIPOLOGÍAS TEXTUALES

Las interacciones entre los diferentes dominios de acción pueden ser extrapoladas a las funciones cumplidas por medios educativos específicos. Por ejemplo, un software diseñado para la visualización y organización del pensamiento, mientras opera principalmente dentro del dominio de la metacognición, puede ser aprovechado también en la resolución de problemas, tanto en las ciencias como en la vida ciudadana.

\subsection{LA SEGUNDA DIMENSIÓN DE LA TAXONOMÍA: TIPOLOGÍAS TEXTUALES}

La clasificación y diseño de cualquier medio educativo conlleva no solamente el establecimiento de sus contenidos y objetivos de aprendizaje, sino también la definición de sus características formales y estructurales. En otras palabras, es necesario identificar cuál es la información relevante que se busca transmitir, y cuál es la manera idónea para hacerlo. Así, mientras que la dimensión de los dominios de acción facilita la comprensión de los objetos y objetivos de los medios educativos, una segunda dimensión debe enfocarse en su forma y estructura. El primer paso para construir esta segunda dimensión consiste en la identificación de los tipos generales de medios con los que se puede abordar la enseñanza del pensamiento crítico.

A pesar de que el concepto de medio, como instrumento para la representación, consumo y registro de información, puede incluir todas las formas de materiales escritos e impresos utilizados tradicionalmente en contextos educativos, a medida que las tecnologías de radiodifusión avanzaron en la segunda mitad del siglo pasado, las nociones de medio educativo comenzaron a enfocarse en otros tipos de material. La imagen de los medios educativos pasó a estar estrechamente vinculada con las películas, la radio y la televisión, y las tecnologías analógicas utilizadas para su aprovechamiento en el aula y el hogar. En los últimos años la popularización de las tecnologías digitales ha ampliado aún más esta noción, trayendo nuevas formas de medios educativos que permiten el aprendizaje interactivo y autoadministrado. Este es el caso de los materiales multimedia y transmedia, los videojuegos y el software dirigido a apoyar tareas específicas en los procesos de aprendizaje.

En esta taxonomía el término de medios educativos engloba a todos los tipos de medio previamente mencionados, disponibles en formatos analógicos o digitales. Estos medios pueden incluir materiales de texto, impresos o digitales, pero se excluyen los medios distribuidos solamente en forma de texto. En ese sentido, materiales escritos como libros de texto y artículos académicos no se consideran objetos para el análisis o diseño a mediante la taxonomía.

La construcción de la segunda dimensión también requiere la identificación de un conjunto de categorías que puedan ser aplicadas de manera consistente al análisis de las características formales de los distintos tipos de medio. Las teorías tradicionales de género no resultan apropiadas para dicha tarea. La teoría de género cinematográfico se centra en convenciones visuales, tales como la escenografía, el vestuario y los atributos físicos de los personajes (Buscombe, 1970), o se enfoca en identidades genéricas que se organizan en paradigmas clásicos (musicales, westerns, películas de gangsters) y fantasías transicionales (terror y ciencia ficción) entre otras categorías (Langford, 2010). En contraste, los géneros televisivos tradicionalmente enfatizan rutinas de producción vinculadas a tipos específicos de programas, tales como las sitcoms, los documentales, los telediarios, los programas de concursos, las series animadas y los programas de telerrealidad. Por otra parte, los videojuegos son clasificados según las características generales de su dinámica de juego, en categorías como acción, deportes, juego de rol, peleas y estrategia entre otras. Los 
materiales multimedia y transmedia, al igual que el software educativo, también pueden ser organizados mediante diversas nomenclaturas.

Estas discrepancias reflejan la naturaleza diversa de los distintos medios educativos e imposibilitan que cualquier teoría de género particular sea utilizada para clasificarlos correctamente. No obstante, se puede afirmar que todos los medios educativos, independientemente del tipo de medio con el que se les identifique, comparten dos características: 1) son, fundamentalmente, textos comunicacionales que buscan transmitir información específica a una audiencia, 2) presentan dicha información mediante estructuras recurrentes, que son (o deben ser) reconocidas y comprendidas por la audiencia.

Una de las aproximaciones más ampliamente conocidas al análisis y clasificación de la estructura de los textos es la teoría de las tipologías textuales. Dependiendo de la naturaleza de la información que se trata de transmitir y de su objetivo de comunicación, cualquier texto puede ser presentado a través de distintas tipologías textuales. En la segunda dimensión de esta taxonomía se introducen cinco categorías de tipología textual (ver tabla 2). Cada una de ellas corresponde a una de las estructuras secuenciales propuestas por Adam (1992): narración, descripción, explicación, argumentación y diálogo.

Tabla 2. Tipologías textuales

\begin{tabular}{|c|c|}
\hline $\begin{array}{c}\text { Tipología } \\
\text { Textual }\end{array}$ & Estructura general \\
\hline Narración & $\begin{array}{l}\text { Los textos narrativos consisten en una secuencia de eventos organizados alrededor de un } \\
\text { marco de tiempo. Una narración requiere la existencia de al menos un personaje que sea } \\
\text { sujeto u objeto de acciones concretas, pero la mayoría de las narraciones incluyen } \\
\text { múltiples personajes que interactúan entre sí. Las secuencias narrativas típicamente } \\
\text { muestran una situación inicial que se convierte en una situación nueva, como resultado } \\
\text { de las acciones de los personajes. }\end{array}$ \\
\hline Descripción & $\begin{array}{l}\text { Mientras los textos narrativos se encuentran estructurados alrededor de eventos y acciones, } \\
\text { los textos descriptivos enfatizan las cualidades de los objetos. Las secuencias descriptivas } \\
\text { suelen incluir una introducción explícita del objeto que va a ser descrito, la identificación } \\
\text { de sus características y partes conformantes, y referencias a su relación con otros objetos, } \\
\text { a través de comparaciones, metáforas y otras figuras retóricas. }\end{array}$ \\
\hline Explicación & $\begin{array}{l}\text { Los textos explicativos organizan información de manera minuciosa, con el fin de } \\
\text { facilitar la comprensión de fenómenos y procesos complejos. Las secuencias explicativas, } \\
\text { explícita o implícitamente, plantean preguntas para luego tratar de ofrecer respuestas. } \\
\text { De esta forma, la explicación generalmente se centra en cómo y por qué algo ocurre. } \\
\text { La explicación se puede usar para presentar definiciones, y relaciones de causa y efecto } \\
\text { o de problema-solución. }\end{array}$ \\
\hline Argumentación & $\begin{array}{l}\text { En contraste con los textos explicativos, que solamente buscan proveer información } \\
\text { concisa sobre un tema, los textos argumentativos pretenden convencer a la audiencia de } \\
\text { la validez de una idea o punto de vista. Los textos argumentativos presuponen la } \\
\text { existencia de ideas o punto de vista contrapuestos. Las secuencias argumentativas } \\
\text { normalmente se encuentran compuestas por el reconocimiento de un tema controversial, } \\
\text { información apoyando algún punto de vista sobre el tema, e inferencias dirigidas hacia } \\
\text { una conclusión. } \\
\text { Algunos textos argumentativos no presentan una secuencia de premisas y conclusiones } \\
\text { para construir un nuevo argumento, sino que se enfocan en el análisis y refutación de un } \\
\text { argumento opuesto. }\end{array}$ \\
\hline
\end{tabular}




\begin{tabular}{|l|l|}
\hline Diálogo & $\begin{array}{l}\text { Su principal característica es la interacción dialógica de dos o más "voces" en la } \\
\text { disposición y presentación de eventos, información o argumentos. Estas voces pueden } \\
\text { ser personajes impulsando el desarrollo de una historia, docentes y estudiantes } \\
\text { interactuando en una clase explicativa o expertos intercambiando argumentos en un } \\
\text { debate. De este modo, los textos dialógicos siempre están estrechamente relacionados } \\
\text { con una o más de las tipologías anteriores. } \\
\text { Al designar al diálogo como una tipología separada se hace posible observar y aprovechar } \\
\text { tipos específicos de interacción, ausentes en otras tipologías cuando éstas se manifiestan } \\
\text { en forma de monólogo. }\end{array}$ \\
\hline
\end{tabular}

Fuente: elaboración propia del autor (2019), basada en Adam (1992).

Es importante anotar que la mayoría de los textos son estructuras jerárquicas y complejas, compuestas por elementos de una o más de las tipologías previamente descritas (Adam, 1992, p. 34). Asimismo, los medios educativos tienden a expresar convergencias de tipologías textuales. Por ejemplo, un programa de televisión sobre los problemas enfrentados por los niños en las escuelas puede utilizar una secuencia explicativa para presentar la naturaleza del problema, y luego usar argumentación y diálogo para mostrar la manera en que los niños trabajan juntos para encontrar una solución.

Figura 2. Taxonomía de medios educativos para el desarrollo del pensamiento crítico

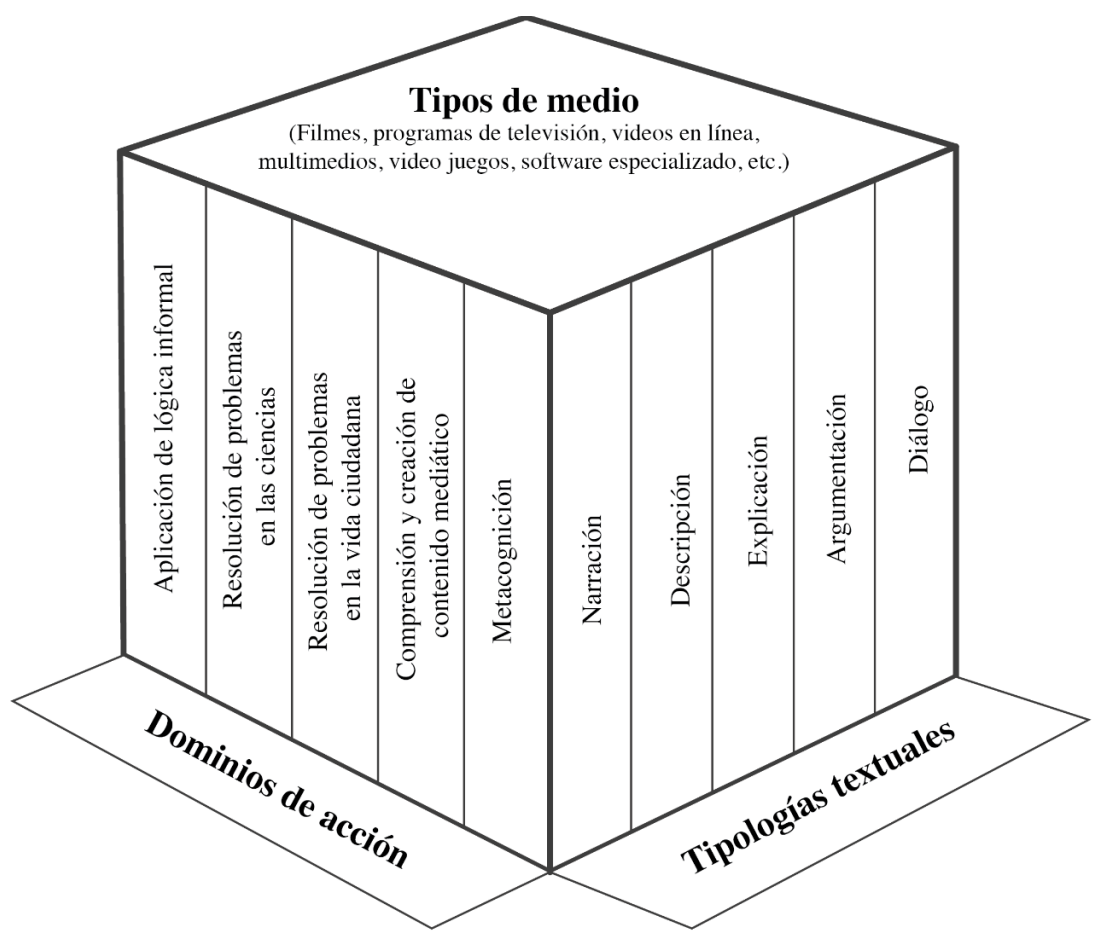

Fuente. Elaboración propia del autor (2019). 


\subsection{CÓMO UTILIZAR LA TAXONOMÍA}

\subsubsection{Análisis y clasificación de medios educativos}

Los dominios de acción presentados en la primera dimensión de la taxonomía facilitan la comprensión de los objetos generales y los objetivos de aprendizaje de los medios dirigidos a promoción del pensamiento crítico. Por otra parte, las tipologías textuales definidas en la segunda dimensión contribuyen en la visualización de las características formales de dichos medios. Al entrar en yuxtaposición, las dos dimensiones de la taxonomía brindan un marco comprehensivo para el análisis, clasificación y diseño de medios educativos (ver figura 2). En esta sección se describen ejemplos concretos de cómo la taxonomía puede ser aprovechada en la realización de estas tareas.

Primeramente, en relación al análisis y clasificación de medios educativos, la taxonomía permite la obtención y sistematización de información que puede ser usada para describir diversos tipos de medios educativos, en una forma breve y consistente. Concretamente, al ser aplicada a cualquier medio educativo, la taxonomía ofrece información sobre qué componente o componentes del pensamiento crítico están siendo abordados, y sobre qué tipologías textuales están siendo utilizadas para abordarlos. Con el objetivo de organizar y enunciar esta información de una manera estandarizada, se propone la siguiente estructura:

\section{Tipo de medio que apoya Dominio de acción usando Tipología textual}

Esta estructura no solamente agiliza la comprensión de la naturaleza de cualquier medio educativo sobre pensamiento crítico, sino que también provee metadatos útiles para su clasificación y organización por parte de investigadores, docentes y administradores de centros educativos. Cinco ejemplos de cómo la taxonomía puede ser utilizada en la clasificación de medios educativos son presentados en la tabla 3. Cada ejemplo corresponde a uno de los distintos dominios de acción.

Tabla 3. Clasificación de medios educativos según la taxonomía

\begin{tabular}{|c|c|c|c|}
\hline $\begin{array}{c}\text { Nombre del } \\
\text { medio educativo }\end{array}$ & Organización & $\begin{array}{l}\text { Tipo de medio y } \\
\text { público meta }\end{array}$ & $\begin{array}{c}\text { Clasificación según la } \\
\text { taxonomía }\end{array}$ \\
\hline $\begin{array}{l}\text { "Mentira la ver- } \\
\text { dad" }\end{array}$ & $\begin{array}{l}\text { Canal Encuen- } \\
\text { tro (Argentina, } \\
\text { 2011-2016) }\end{array}$ & $\begin{array}{l}\text { Es un programa de televisión } \\
\text { dirigido a jóvenes y adultos, } \\
\text { que presenta monólogos sobre } \\
\text { la religión, la felicidad, la belle- } \\
\text { za, la justicia y otros temas. Es- } \\
\text { tos monólogos se encuentran } \\
\text { embebidos en una serie de } \\
\text { eventos que involucran a diver- } \\
\text { sos personajes (Encuentro, Sin } \\
\text { fecha). }\end{array}$ & $\begin{array}{l}\text { Un programa de televisión } \\
\text { que apoya la aplicación de } \\
\text { lógica informal usando ar- } \\
\text { gumentación y narración. }\end{array}$ \\
\hline
\end{tabular}




\begin{tabular}{|c|c|c|c|}
\hline "Quandary" & $\begin{array}{l}\text { Learning games } \\
\text { network } \\
(\text { EEUU, 2012) }\end{array}$ & $\begin{array}{l}\text { Es un video juego dirigido a es- } \\
\text { tudiantes de primaria y secun- } \\
\text { daria que presenta historias en } \\
\text { las que un grupo de colonos se } \\
\text { enfrentan a problemas en un } \\
\text { planeta lejano. El usuario tiene } \\
\text { el rol de guiar la discusión y } \\
\text { ayudar a los personajes a en- } \\
\text { contrar una solución al proble- } \\
\text { ma (Learning games network, } \\
\text { 2012). }\end{array}$ & $\begin{array}{l}\text { Un video juego que apoya } \\
\text { la resolución de problemas } \\
\text { en la vida ciudadana usan- } \\
\text { do narración y diálogo. }\end{array}$ \\
\hline 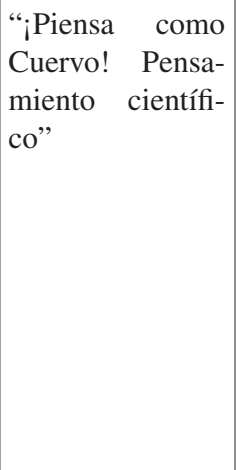 & $\begin{array}{l}\text { NHK (Japón, } \\
\text { 2013). }\end{array}$ & $\begin{array}{l}\text { Es un programa de televisión } \\
\text { para estudiantes de primaria. } \\
\text { Presenta preguntas sobre fenó- } \\
\text { menos naturales y luego deja } \\
\text { que sean los televidentes quie- } \\
\text { nes busquen las respuestas por } \\
\text { su propia cuenta. Los episodios } \\
\text { del programa están disponibles } \\
\text { en línea, junto con las respues- } \\
\text { tas ofrecidas por los televiden- } \\
\text { tes y con materiales comple- } \\
\text { mentarios (NHK, Sin fecha). }\end{array}$ & $\begin{array}{l}\text { Un programa de televisión } \\
\text { que apoya la resolución de } \\
\text { problemas en las ciencias } \\
\text { usando explicación y des- } \\
\text { cripción. }\end{array}$ \\
\hline "The cleaners" & $\begin{array}{l}\text { Gebrueder } \\
\text { Beetz } \\
\text { Filmproduktion, } \\
\text { (Alemania, } \\
2018 \text { ) }\end{array}$ & $\begin{array}{l}\text { Es un filme de educación conti- } \\
\text { nua que discute sobre la proli- } \\
\text { feración de noticias falsas y vi- } \\
\text { siones radicalizadas en el esce- } \\
\text { nario global, y expone sobre las } \\
\text { organizaciones que trabajan } \\
\text { para "limpiar" dichos conteni- } \\
\text { dos en la web (Gebrueder Beetz } \\
\text { Filmproduktion, 2018). }\end{array}$ & $\begin{array}{l}\text { Un filme que apoya la com- } \\
\text { prensión y creación de } \\
\text { contenido mediático usan- } \\
\text { do explicación y argumen- } \\
\text { tación. }\end{array}$ \\
\hline $\begin{array}{l}\text { "L o i l o N o t e } \\
\text { School" }\end{array}$ & $\begin{array}{l}\text { Loilo (Japón, } \\
\text { 2014) }\end{array}$ & $\begin{array}{l}\text { Es un sistema de aprendizaje } \\
\text { colaborativo mediado por com- } \\
\text { putadora (CSCL), dirigido } \\
\text { principalmente a escuelas pri- } \\
\text { marias y secundarias. Facilita la } \\
\text { creación de presentaciones y el } \\
\text { uso de tarjetas para expresar, } \\
\text { organizar y compartir ideas en } \\
\text { escritorios virtuales (Loilo, Sin } \\
\text { fecha). Una versión reciente in- } \\
\text { cluye organizadores gráficos } \\
\text { que permiten la visualización } \\
\text { de procesos cognitivos (Ku- } \\
\text { rokami \& Kojima, 2018). }\end{array}$ & $\begin{array}{l}\text { Un sistema de CSCL que } \\
\text { apoya la metacognición } \\
\text { usando explicación y des- } \\
\text { cripción. }\end{array}$ \\
\hline
\end{tabular}




\subsubsection{Diseño de medios educativos}

En relación al diseño de medios educativos para el desarrollo del pensamiento crítico, la taxonomía ofrece una guía para apoyar dicho proceso. Dependiendo de las condiciones en las que los medios educativos son creados, es posible sugerir dos procedimientos distintos. El primero es para aquellos casos en los que el diseño inicia con la búsqueda de una solución para un problema educativo. El segundo se propone para aquellos casos en las que el proceso de creación inicia como un esfuerzo técnico o artístico.

Idealmente, la creación de cualquier medio educativo inicia como una respuesta al reconocimiento de una necesidad educativa, como una reacción ante la identificación de objetivos de aprendizaje concretos que no están siendo debidamente atendidos. En estos casos, como un primer paso, los creadores pueden remitirse a la dimensión de dominios de acción, de manera que puedan visualizar el componente o componentes del pensamiento crítico relacionados con los objetivos de aprendizaje a ser abordados. Una vez que los dominios de acción han sido definidos, y con base en ellos se ha establecido una idea general de los contenidos a tratar, los creadores pueden proceder a la selección de las tipologías textuales pertinentes para la presentación de dichos contenidos. En este punto, es posible tomar decisiones informadas sobre los tipos de medio que pueden apoyar de mejor manera el cumplimiento de los objetivos de aprendizaje.

El proceso de diseño puede visualizarse fácilmente utilizando la representación gráfica de la taxonomía (ver figura 2) como una "caja de diseño". Los creadores de los medios educativos pueden iniciar enfocándose en el lado izquierdo de la caja, mientras mantienen un ojo en el lado derecho y el lado superior. Luego pueden trasladar la atención al lado derecho, cotejando con el lado izquierdo las veces que sea necesario, hasta obtener una imagen clara del tipo de medio idóneo a ser producido. Por último, pueden usar los resultados del proceso para seleccionar alguna de las opciones disponibles en el lado superior de la caja.

En los casos en los que los creadores son expertos en tipos específicos de medio, produciendo los medios en los que tienen experticia ya sea por su propio interés personal o porque han sido contratados para hacerlo, el proceso de diseño debe abordarse desde la dirección opuesta: de arriba hacia abajo. Debido a que el tipo de medio ha sido definido antes que los dominios de acción, es posible tomar dos rutas en el proceso de diseño.

La primera ruta supone un tránsito "arriba-izquierda-derecha" dentro de la caja de diseño. Este camino se recomienda para aquellos casos en los cuales los creadores tienen completa libertad para seleccionar los dominios de acción y las tipologías textuales con los que desean trabajar. Pueden iniciar desde el lado superior y luego bajar por el lado izquierdo de la caja, para definir los dominios de acción, y por añadidura, el conjunto de objetivos de aprendizaje específicos del medio educativo. Pueden luego seguir el procedimiento de cotejar repetidamente los dominios de acción y las tipologías textuales, hasta encontrar una combinación adecuada.

La segunda ruta conlleva un tránsito "arriba-derecha-izquierda" y se recomienda para aquellos casos en los que características adicionales de los medios educativos han sido determinadas previamente, poniendo limitaciones sobre el uso efectivo de una o más de las tipologías textuales. Por ejemplo, si una televisora solicita a los creadores la producción de una serie animada dirigida a niños de preescolar, desde el punto de vista del desarrollo cognitivo de los miembros del público meta, se pueden esperar limitaciones en el uso de 
la explicación y la argumentación. Para estos casos se recomienda descender de la caja de diseño deteniéndose primero en su lado derecho, para lograr una comprensión completa de las características formales a las que el medio educativo va a tener que ajustarse. Seguidamente los creadores pueden fijar su atención en el lado izquierdo de la caja para determinar los dominios de acción idóneos para ser tratados en el medio educativo en cuestión.

\section{DISCUSIÓN Y CONCLUSIONES}

La taxonomía presentada en este artículo se propone como una herramienta para la convergencia fluida y provechosa de educadores, investigadores y creadores, que trabajan conjuntamente tanto en la producción de medios educativos para el desarrollo del pensamiento crítico, como en su análisis y clasificación. A través del planteamiento de la taxonomía también se abren espacios para reflexionar sobre tres temas vinculados a la concepción pensamiento crítico y a la forma en que éste se relaciona con los medios educativos.

Primeramente, se resalta que debido a la naturaleza multidimensional de lo que típicamente se considera como pensamiento crítico, es extremadamente difícil abordarlo en su totalidad con un solo medio educativo. Asimismo, se puede afirmar que esa intención puede llevar a la producción de medios educativos carentes de abordajes metodológicos y objetivos de aprendizaje claros. En ese sentido, a la hora de promover el pensamiento crítico a través de los medios educativos, ya sea en la educación escolar o en la educación permanente, se recomienda recurrir a un uso coordinado de distintos medios, de manera que cada uno de ellos cumpla un rol concreto en el abordaje de componentes específicos del pensamiento crítico, al mismo tiempo que complementa a los otros.

Segundo, se reconoce que cualquier esfuerzo para comprender y clasificar los medios educativos no puede escapar las controversias históricas en la investigación sobre el pensamiento crítico. Este es el caso de las discusiones relacionadas con la transferencia de habilidades de pensamiento crítico a través de distintos dominios de conocimiento, y la efectividad comparada de diferentes formas de instrucción y práctica, tales como el juego de roles, el uso de narrativa y la experimentación. La manera en que la taxonomía puede contribuir al desarrollo de estas discusiones dentro del campo de los medios educativos se plantea como el objeto de investigaciones ulteriores.

Finalmente, es importante delimitar el alcance de la taxonomía, y explicitar los criterios generales para identificar los medios idóneos para ser diseñados y clasificados con la misma. Se deben diseñar y clasificar medios educativos que traten al pensamiento crítico como un instrumento útil para la selección y realización de acciones significativas dentro del marco delineado por la taxonomía. En otras palabras, se deben abordar solamente los medios educativos enfocados en formas de pensar, y en las acciones basadas en dichas formas de pensar, excluyendo a los medios limitados a la presentación de información, sin pistas o instrucciones claras sobre cómo adquirir o aplicar las habilidades de pensamiento crítico. 


\section{REFERENCIAS BIBLIOGRÁFICAS}

Adam, J. (1992). Les textes: Types et prototypes: Recit, description, argumentation, explication et dialogue. Nathan.

Binkley, M., Erstadt, O., Herman, J., Raizen, S., Miller-Ricci, M. \& Rumble, M. (2012). "Defining Twenty-First Century Skills" in Griffin, P., McGaw, B. and Care, E. (Eds.) Assessment and Teaching of $21^{\text {st }}$ Century Skills. Springer, 17-66.

Buscombe, E. (1970). The Idea of Genre in American Cinema. Screen, 11(2), 33-45.

Carvalho, D., Azevedo, I., Cruz, G., Mafra, G., Rego, A., Vitor, A., . . Ferreira, M. (2017). Strategies used for the promotion of critical thinking in nursing undergraduate education: A systematic review. Nurse Education Today, 57, 103-107. doi: 10.10164/j.nedt.2017.07.010.

Chan, Z. (2016). A systematic review on critical thinking in medical education. International Journal of Adolescent Medicine and Health, 30(1), 1-11. doi:10.1515/ijamh-2015-0117.

Csapó, B. (Ed), \& Funke, J. (Ed). (2017). The nature of problem solving: Using research to inspire 21st century learning. Paris: OECD. doi:10.1787/9789264273955-en.

Dwyer, C., Hogan, M., \& Stewart, I. (2014). An integrated critical thinking framework for the 21 st century. Thinking Skills and Creativity, 12, 43-52. doi: 10.1016/j.tsc.2013.12.004

Dewey, J. (1910). How we think. Dover.

Eisenberg, M., Lowe, C., \& Spitzer, K. (2004). Information literacy: Essential skills for the information age (2nd ed.). Libraries Unlimited.

Encuentro (Sin fecha). Catálogo. Última visita 20 de mayo de 2019. Recuperado desde: http:// encuentro.gob.ar/programas/serie/8023

Ennis, R. (2016). Critical thinking across the curriculum: A vision. Topoi, 37(1), 165-184. doi:10.1007/s11245-016-9401-4

Facione, P. (1990). Critical thinking: A statement of consensus for purposes of educational assessment and instruction. The American Philosophical Association Delphi report. Millbrae: California Academic Press.

Gebrueder Beetz Filmproduktion. (2018). The cleaners. Última visita 9 de setiembre de 2020. Recuperado desde: https://gebrueder-beetz.de/en/productions/the-cleaners

Griffin, P., McGaw, B. and Care, E. (2012) Assessment and Teaching of $21^{\text {st }}$ Century Skills. Springer

Halpern, D. (2003). Thought and Knowledge: An introduction to Critical Thinking (Fourth Edition). Lawrence Erlbaum.

Hennessey, M. (1999). Probing the dimensions of metacognition: Implications for conceptual change teaching-learning. Recuperado desde: https://files.eric.ed.gov/fulltext/ED446921.pdf

Higuchi, N. (2012). Tendencias y problemas en la investigación del pensamiento crítico en Japón: Énfasis en la pedagogía. Investigación de métodos educativos, 17, 199-225.

Hincapie, D., Ramos, A., y Chirino, V. (2018). Aprendizaje basado en problemas como estrategia de aprendizaje activo y su incidencia en el rendimiento académico y pensamiento crítico de estudiantes de medicina. Revista Complutense De Educación, 29(3). 665-681. doi:10.5209/ RCED.53581

Johnson, R. (2014). Rise of informal logic. Centre for Research in Reasoning, Argumentation and Rhetoric (CRRAR): Windsor Studies in Argumentation. doi: 10.22329/10.22329/wsia.02.2014.

Krathwohl, D. (2002). A revision of bloom's taxonomy: An overview. Theory into Practice, 41(4), 212-218. doi:10.1207/s15430421tip4104_2.

Kurokami, H. \& Kojima, A. (2018) Development and Effectiveness of Digital Graphic Organizers. International Journal for Educational Media and Technology. 12(1), 57-64.

Kusumi, T. (Ed.), y Michita, Y. (Ed.) (2015). Pensamiento crítico: Fundamento de las literacidades para sobrevivir en el siglo XXI. Shinyosha.

Kusumi, T. (Ed.), y Michita, Y. (Ed.) (2016) Pensamiento crítico y literacidad ciudadana: Habilidades del siglo XXI para cambiar la educación, los medios y la sociedad. Seishin Shobo. 
Langford, B. (2010). Film Genre: Hollywood and Beyond. Edinburg University Press.

Learning games network (2012). Quandary. Última visita 9 de mayo de 2019. Recuperado desde: https://www.quandarygame.org/

Lewis, A., \& Smith, D. (1993). Defining higher order thinking. Theory into Practice, 32(3), 131-137. doi:10.1080/00405849309543588.

Loaiza, Y., y Osorio, L. (2018). El desarrollo de pensamiento crítico en ciencias naturales con estudiantes de básica secundaria en una institución educativa de Pereira - Risaralda. Diálogos Sobre Educación. Temas Actuales En Investigación Educativa, 9(16). Recuperado desde: http:// www.scielo.org.mx/pdf/dsetaie/v9n16/2007-2171-dsetaie-9-16-00009.pdf

Loilo (Sin fecha). Loilo Note School. Última visita 11 de setiembre de 2020. Recuperado desde: https://n.loilo.tv/en/

Marzano, R. \& Kendall, J. (2007). The new taxonomy of educational objectives (2nd ed.). Corwin Press.

Mayer, R. (1992). Thinking, problem solving, cognition (2nd ed.). W.H. Freeman.

Michita, Y. (2008). Profundización del pensamiento crítico mediante los mecanismos de la metacognición. Espíritu de la Modernidad, 497, 59-67

Newman, F. (1990). Higher order thinking in teaching social studies: A rationale for the assessment of classroom thoughtfulness. Journal of Curriculum Studies, 22, 41-56.

NHK (Sin fecha). NHK for school. Última visita 9 de enero de 2019. Recuperado desde: http://www. nhk.or.jp/rika/karasu/

Nickerson, R. (1984). Kinds of thinking taught in current programs. Educational Leadership, 42(1), 26-36.

Ministry of Education of Ontario (1989). Media Literacy Resource Guide: Intermediate and Senior Division. Ministry of Education of Ontario.

Paul, R., Binker, A., \& Weil, D. (1990). Critical Thinking Handbook: K-3. Foundation for Critical Thinking, Sonoma State University.

Resnick, L. (1987). Education and learning to think. National Academy Press.

Rodríguez, A. (2018). Elementos ontológicos del pensamiento crítico. Teoría De La Educación. Revista Interuniversitaria, 30(1), 53-74. doi:10.14201/teoredu3015374

Rychen, D. \& Salganik, L. (2003). Key Competencies for a Successful Life and a Well-Functioning Society. Hogrefe Publishing

Santos, L. (2017). The role of critical thinking in science education. Journal of Education and Practice, 8(20), 159-173. doi:10.1023/A:1016042608621

Siew, N. \& Mapeala, R. (2016). The effects of problem-based learning with thinking maps on fifth graders' science critical thinking. Journal of Baltic Science Education, 15(5), 602-616.

Swartz, R., Costa, A., Beyer, B., Reagan, R. \& Kallick, B. (2010). Thinking-Based Learning: Promoting Quality Student Achievement in the 21st Century. Teacher college press.

Widyatiningtyas, R., Kusumah, Y. S., Sumarmo, U., \& Sabandar, J. (2015). The impact of problembased learning approach to senior high school students' mathematics critical thinking ability. Indonesian Mathematical Society Journal on Mathematics Education, 6(2), 30-38. 